\title{
Web 2.0 revisited: user-generated content as a social innovation
}

\section{Christoph Kaletka and Bastian Pelka*}

\author{
Sozialforschungsstelle Dortmund (sfs), \\ Central Scientific Institute of the Technische Universität Dortmund, \\ Evinger Platz 17, Dortmund 44339, Germany \\ Email: kaletka@sfs-dortmund.de \\ Email: pelka@sfs-dortmund.de \\ *Corresponding author
}

\begin{abstract}
This paper raises the question whether Web 2.0 can be seen as a technological or a social innovation and which interdependencies exist between these two innovative aspects of the phenomenon. For that purpose, the definition of Web 2.0 as a tag cloud (for example given in Wikipedia) or as a difference in comparison to a 'Web 1.0' is revisited, challenged and discarded. In following steps, the paper argues that the core innovation of Web 2.0 is the communication of 'user-generated content' as a new social routine. The main enabling factors for Web 2.0 utilisation as a social routine are identified as easy-to-use software and broadly spread internet access. So while technology is seen as a 'catalyst' of the phenomenon, the innovation itself (user-generated content) is considered a social one.
\end{abstract}

Keywords: Web 2.0; social web; social innovation; user-generated content; wiki; blog; community.

Reference to this paper should be made as follows: Kaletka, C. and Pelka, B. (2011) 'Web 2.0 revisited: user-generated content as a social innovation', Int. J. Innovation and Sustainable Development, Vol. 5, Nos. 2/3, pp.264-275.

Biographical notes: Christoph Kaletka is a Coordinator of research unit 'Labour and Education in Europe' at Sozialforschungsstelle Dortmund, Central Scientific Institute of the Technische Universität Dortmund, Germany. He received his $\mathrm{PhD}$ in Communication Science on 'political internet fora'. His main fields of work are (European) labour and employment policy, regional development, the transition from school to the world of labour, VET research and consultation and political communication.

Bastian Pelka is a Coordinator of research unit 'Labour and Education in Europe' at Sozialforschungsstelle Dortmund, Central Scientific Institute of the Technische Universitat Dortmund, Germany. He received his $\mathrm{PhD}$ in Communication Science on 'artificial intelligence as communication form'. His main fields of work are vocational training in the European context, ICT, 'Web 2.0' and other media, especially e-Learning. A current research and advisory topic is the transition from school to the world of labour. He is a Lecturer at different universities in ICT-linked topics. 


\section{Background: what is 'Web 2.0'?}

The 'Web 2.0' is widely seen as one of the most important recent and most influential innovations in the field of ICT. It is regarded as an innovation itself and a place that again bears media innovations (such as Wikipedia, YouTube or Flickr). The annual conference of the German Society for Communication Science (DGPuK) impressively illustrated the impulse that experts see in this field: around $80 \%$ of all papers delivered under the headline 'media innovations' (see www.dgpuk.de/) addressed Web 2.0. In the ICT discourse, Web 2.0 is often seen as a new technology that represents an innovation compared to the 'Web 1.0'. This understanding condensates in the approach to define Web 2.0 by collecting all applications that are declared to be Web 2.0. This self-reflexive definition is expressed in the tag cloud that Wikipedia uses to describe Web 2.0 (see http://en.wikipedia.org/wiki/Web_2.0). The tag cloud implies concrete applications (wikis and blogs), principles (modularity and joy of use), technologies (AJAX and RSS) and utilisation phenomena (long tail, folksonomies and user-generated content). The tag cloud indicates one of the basic problems of all discourses on Web 2.0: The phenomenon is difficult to define, as it implies a lot of different layers (applications, principles, technologies and utilisation) and on the other side does not have a sharp cornerstone to a 'Web 1.0'. This leads to the question if Web 2.0 is really an innovation. And - if yes which kind of innovation it is and what relationship technology and social aspects take.

\section{Approach: two innovation layers}

If we follow Reißmann (2005) that technology and software that are widely understood as 'Web 2.0' existed a long time before we came to that name and follow Maaß and Pietsch (2007) that most of the Web 2.0 applications can be seen as varieties of older applications which have developed over decades (Rheingold, 1992; Bühl, 1997), we must deny that Web 2.0 could be a technological quantum jump. Indeed, there is no step of version for a single technology that could justify the new version number. At the same time, the word 'Web 2.0' sums up a lot of aspects amalgamating to a phenomenon that seems worth to be called 'new' in comparison to a world without them. Against this background feeling, this paper seeks for the core innovation of the phenomenon called 'Web 2.0'. Once found, the core innovation has to be defined and analysed by the instruments of innovation research. After having denied that Web 2.0 is a technological innovation, we must open the focus and look for alternative innovation layers.

The scientific discourse on 'media' has long since introduced an important distinction: it differentiates between technological and social, or 'first layer' and 'second layer' media (Kubicek, 1997, p.33). For a coherent use of technologies in a society, 'technologies' have to be embedded into 'media' which institutionalise the way technologies are being used and set the 'rules of the game' (Wirth and Schweiger, 1999, p.46). In this understanding, 'media' is understood as a social layer of mediated communication. It describes the social aspects of creation and distribution of communication, such as professional roles, economic frames and institutionalisations of communication, and is distinguished from the used technology base - such as printing, television and radio broadcast or online access. This understanding seems adequate to identify the innovation within the phenomenon 'Web 2.0 '. 
So, in line with this argumentation, we will distinguish the two layers - social and technological - of Web 2.0. Therefore, we take a closer look on two applications (wikis and blogs) that are seen as part of the Web 2.0 to examine their innovative character. For wikis and blogs, we distinguish between software (the technology layer), content (the mediated communication), communication situation (many-to-many, few-to-many and one-to-many) and institutionalised utilisation in social routines. The latter describes the 'rules of the game' for an interplay of software and content under which users cooperate, communicate and interact.

\section{Analysis of two examples}

\section{I Wiki}

A wiki is an online encyclopaedia in which content is being created, altered and discussed cooperatively by different authors. Here it is important to make a distinction between software, content and application of a wiki (Pelka, 2008). This distinction is viable for all Web 2.0 media, as the following examples will illustrate.

The first layer of analysis we call 'software'. It is the basic technology structure of the wiki and is administrating content plus offering the user a number of options to interact with the database as well as with other users. It can be used to gather content, discuss and publish it. The user will notice this technology only as an interface to contents and as a platform for interaction. For wikis, we can observe the aim to reduce the time the user has to spend on learning how to use the software and use it at all. So, in wikis, software is intended to step to the background of the users view and leave the carpet for 'content'.

'Content' - the second layer of our analysis, which in case of a dictionary means articles, links, media and also communication in discussion threads of related fora - is being created by the users themselves. Unlike traditional dictionaries and encyclopaedia, most wikis do not have an editorial office, so there are complementary options to discuss texts. Again, the users serve as editors (Pelka et al., 2008). This implies new demands on the structures of content production. While traditional dictionaries gain their content out of professional structures and can rely on professional mechanisms, wikis must create new mechanisms, for example for quality assurance and agenda setting. This change of paradigms - from central responsibility with clear agendas and professional instances of content production to distributed content production - is not only causing conflicts between the systems - like the discussion between Wikipedia and Encyclica Britannica on quality issues - but is also giving birth to new actors and actor constellations. This change of paradigms is currently challenging structures of content production in different media, professional systems and even parts of society.

Our third layer of analysis refers to the interplay of the two above mentioned. In our analysis, the term 'application' refers to the interaction between software and content, as we argue that the interplay produces a new instance of opportunities and innovation itself. This interaction leads to a new realm of options which is crucial for the process of creating content. For example, the possibilities offered by the software 'MediaWiki' (which is the technology that Wikipedia uses), are only exploited within the context of a specific content, as the discussion facilities offered by MediaWiki are only used in 
concrete linkage to a specific content. So a wiki combines an inventory of knowledge with supporting options for authors to cooperate and communicate and therefore create additional content.

The best known wiki phenomenon is certainly the 'Wikipedia'. Its most striking characteristics are the extent and the quality. Wikipedia in German language comprises about 1.2 million articles and the English version about 3.4 million (numbers from September 2010). It works on the basis of a small number of rules and a decentralised quality management with users ensuring that these rules are being followed. The comprehensive use of Wikipedia on a high frequency by a large number of users/authors can be considered the factor of success in this case. If you look at pure numbers, the future looks bright for Wikipedia. An online study on media use conducted by ARD and ZDF asked for Web 2.0 media. Among the 14- to 19-year-old, $82 \%$ used Wikipedia in 2007 - in 2009, 91\%. Considering these developments, a few differences between a wiki and the comparable offline equivalents are visible: the wiki gains its content and therewith connected its quality and reliability out of the users. This anonymous, heterogeneous and non-professional way of producing content clearly distinguishes it from the structured process of content production within the professional environment of - for example - a publishing house.

For this reason, we come to the conclusion that the core innovation of a wiki is the enabling and supporting of the creation of content by users - user-generated content.

\subsection{Blog}

A blog - a made-up word combining the terms 'Web' and 'log' - is an internet-based diary. The internet tracing service 'Technorati' has recently counted over 80 million blogs worldwide. Certainly, these show the most heterogeneous quality possible comprising professionally designed and funded blogs as well as once installed but meanwhile abandoned 'ruins'.

Again, we have to distinguish between software, content and the application of blogs. The software establishes an interface between users and content and allows users to publish and administrate content. In a way blog software is the prime example for increasing the number of users and authors through technical simplicity. Meanwhile, the Microsoft Office software offers a button to publish an Office-document on a blog. The result is: everybody able to handle the Word programme on a minimum level can publish a blog. The technology ('software') steps back and is not visible to the user. At least, the user faces very low technological barriers in using a blog.

The 'content' of a blog are the articles written by the user. A blog consists of an index page displaying the most recent post on top. New posts make older ones move further down. So theoretically a blog is an infinite line of entries, comprises links and search functions and can therefore serve as an archive. An entry, or a 'post', consists of a headline and a short introductive text (teaser) and a hyperlink to the full text entry. In many cases, the teaser is generated automatically from the first lines of the full text.

This way, blogs waive the function of newspaper teasers which deliberately advertise full texts on the following pages. While newspaper teasers are written by the editorial staff and therefore represent a selection outcome of professionally trained and institutionally bound editors (Weischenberg, 1990, p.59ff.), a blog teaser is a mere copy of the 
first words of the blog. It is exactly this institutionalised selection process and decision making by a third party (the editorial staff in case of newspapers) which blogs do not include: They disclaim editorial standards to a large extent.

What is the innovation of blogs? Due to the fact that every post has a unique and unchangeable address, bloggers can refer to these entries by setting 'permalinks'. Intensive permalinking of numerous blogs has created a vast network of references: the 'blogosphere'. By linking blogs and blog entries quick and easily, authors can provide news to a considerable public in a short period of time. The blogosphere can be seen as a new public being held together by technology and the shared interested in the discussed topics by users that create the content of the blogosphere themselves.

Neuberger et al. (2007, p.96) see in blogs the "transition from editorially driven publishing to participation". Like wikis are edited by users and not professional editors in publishing houses, blogs are edited by users instead of professional journalists working for a newspaper. This raises the question of the different production, selection and publishing strategies of both systems. Neuberger et al. paid special attention to the potential of blogs in terms of agenda setting. 'Agenda setting' describes the ways in which topics are selected for publication by the media. An impressive example for agenda setting of a Web 2.0-based social movement is the 'San Precario' movement in Spain and Italy. Political activists invented 'San Precario' as the patron saint of all precariously employed people and published numerous blogs around him. Speed, simplicity and low costs of this medial approach supported the agenda setting and helped create a Europe-wide network of activists. Their impact was not limited to digital media. Many TV stations and newspapers reported the story and, in a reflexive process, delivered topics which were discussed in the blogosphere again.

Leskovec et al. (2009) showed that there is a strong linkage between the blogosphere and traditional newspapers - they both refer to each other and form a discourse which impacts on the agenda setting of a society. The same study also showed that traditional media and the blogosphere use different selection criteria to decide which information becomes a 'news' and which not (in other words: which information is published by the two systems). The blogosphere is too young and still moving too fast to set final distinctions in selection criteria. However, it seems that blog authors gain their topics both from the blogosphere and traditional media, while traditional media are more selfreflective - in other words: they tend to refer to other traditional media rather than the blogosphere. Also there seem to be differences between both 'media' with respect to the speed of communication and the form of storytelling.

\subsection{Conclusion: the perspective of social innovation}

IT-based networks or communities are not an invention of Web 2.0. They can even be traced back to the time when the internet was not yet public (Rheingold, 1992). Dery (1996, p.29ff.) considered even these early communities mainly as a technological prolongation of the 1960s' discourse culture, which in itself was socially innovative. These approaches addrèss one of the central questions of the present article: if Web 2.0 is regarded as something new, but is not a technological innovation, what is the innovation? Howaldt et al. (2009) introduce a fruitful perspective by differentiating between a technological and a social layer of innovation and ascribing the knowledge society a fundamental change in the relation between these two layers. The transition from industry to knowledge society corresponds with a change of paradigm in the innovation 
system. Within this change, the relationship between social and technological innovations is changing (Howaldt et al., 2008). Social innovations are gaining impact in comparison to technological innovations.

Howaldt and Schwarz (2010, p.21) define with a reference to Schumpeter:
"A social innovation is new combination and/or new configuration of social practices in certain areas of action or social contexts prompted by certain actors or constellations of actors in an intentional targeted manner with the goal of better satisfying or answering needs and problems than is possible on the basis of established practices. An innovation is therefore social to the extent that it, conveyed by the market or 'non/without profit', is socially accepted and diffused widely throughout society or in certain societal sub-areas, transformed depending on circumstances and ultimately institutionalized as new social practice or made routine."

With this definition, Howaldt and Schwarz do not only separate a social innovation from technological innovations, but also from social inventions and social change. Social inventions are intended, new and social, but not used. And social change is not intended.

By taking the perspective of social innovation, we can sharpen our view on the phenomenon Web 2.0. This perspective allows us to focus on the social aspects of the Web 2.0 and made us follow Howaldt and Schwarz's definition parts of a social innovation: intangibility, novelty, concrete adoption in the practice and intendedness. By taking this perspective, we come to identify three new social aspects regarding Web 2.0 services:

1 the simplicity aspect of generating, administrating, and publishing content that enables concrete usage by a broad and heterogeneous audience (Kaletka and Pelka, 2010)

2 the quantitative aspect: Web 2.0 communities are no longer restricted to people with a high affinity for IT but well-known meeting-points 'for the masses' with their main interest not in IT

3 the qualitative aspect: a motivation for being a part and an active member of the community is the social prestige that comes with it (for an introduction to Webbased social networking and Web 2.0 in the field of adult education, see Diaz and Rissola, 2009, p.250ff.). This makes clear that Web 2.0 is intended - at least for some actors - and does not 'happen'.

Seeing these together, Web 2.0 is a social innovation that has the potential to influence the way people work, communicate and participate. The result is an emerging network of users who have built relations among each other, because of common interests or information needs, and who are also willing to publish and share information to a certain degree. Or, with Wassermann and Faust:

"A social network consists of a finite set of actors and the relation or the
relations defined on them. The presence of relational information is a critical
and defining feature of a social network." (Wasserman and Faust, 1994, p.20)

The core innovation of blogs seems to be the effortlessness of publishing. Users can use blogs to publish information with a low level of ICT skills. Combined with low costs for internet access, a broader number of users are able to blog. This of course is only true for countries with good ICT infrastructure and competences, but the example of blogging Chinese or Arabian youth shows that especially the low technical and skill demands enable the publishing of communication outside of traditional media structures. 
This first analysis of wikis and blogs leads us to the interim result that these applications are a new way of using 'old' technology in order to create content outside of professional routines and practices (such as journalism) to make it available via internet. The application of such Web 2.0 services catalyses new 'rules of the game': social routines which satisfy expectations of user-generated content.

From this point on, we argue that this user-generated content as a new social routine is the core innovation that best describes the innovative character of Web 2.0. For this reason, it is important to define Web 2.0 by its non-technical characteristics. We regard user-generated content as the unique feature of Web 2.0. The OECD report 'Participative web: user created content' has taken an approach which is in various aspects congruent with the authors' understanding of Web 2.0. According to their definition, the participative web consists of "(i) content made publicly available via the Internet, (ii) which reflects a 'certain amount of creative effort', and (iii) which is 'created outside of professional routines and practices"'.

If, as said above, such user-generated content is really created outside professional structure, but still communicated within easily understandable social routines, such content poses a challenge for professional selection instances and journalism as a whole in the same way book printing challenged the role of the church as dominating gatekeeper in the religious discourse centuries ago.

As already indicated above, there are certain prerequisites which have to be met for the emergence of social innovations which depend on technology. Here, as Howaldt and Schwarz $(2010, p .3)$ said, the "preparedness of society to adopt new solutions for needs and challenges comes into play. (...) Social values, ideologies, institutions, power imbalances, other disparities, and - last but not least - prevailing patterns of innovations have an effect on the success of different kinds of innovation ("path dependency')." One important factor of preparedness is the extent of media use in a society. In recent years, the use of the internet has increased tremendously in Germany. While in 1997, about 5 million people accessed the internet, this number increased to 35 million in 2003 and 43 million in 2008 (ARD/ZDF online study 2009). At the same time, the socio-demographic characteristics have changed: users more and more represent the overall population, with deviations especially related to the age of users. Young people still use the internet to a much higher degree than elderly people as well as some target groups with social disadvantages. This divide is being addressed through political action and NGO initiatives for digital inclusion. One current example is the initiative 'Internet erfahren' ('Experience the internet') by the German Federal Department of Economics and Technology which takes a promising approach by involving different groups of mediators, multipliers and facilitators who directly help senior citizens take their first steps online.

In short: Today, there are more people online, and also more people who have less experience in technology use. This major trend is an important element of the emergence of Web 2.0 as a social innovation, because many applications are directly dependant on a large user group. So we can argue that the innovation introduced by 'Web 1.0', namely the internet eruption in the public sphere around 1995, which was based on allowing user interaction with content producers and broadcasters (websites) and other users (chats and emails) foremost bilaterally, prepared the field for the emergence of Web 2.0 characterised by content generated by users but with the main purpose of sharing it with peers, giving room to a culture of collaboration and speeding up related processes such as the open source movement and a new discussion of property rights regulations. 
As the examples given above have shown, the fundamental principles of communication in Web 2.0 are different from mass media communication. While letters to the editor used to be one of the very few options to initiate something like a dialogue in mass media, users generate Web 2.0 content on a regular basis. Or, with Alby (2007, p.11): "The system requirements for Web 2.0 were the development of a user 2.0, which again required data transfer 2.0 and access costs 2.0 ."

\section{Practical use and results of this perspective change}

This perspective on the subject 'Web 2.0' also allows a new look on the discussion of technologies and their evolution. Especially, it helps us finding answers not only to the question which prerequisites and rules are fundamental for specific user-generated content as a social innovation, but also to the question of how Web 2.0 applications may be intentionally used in different fields of practice.

Therefore, we will return to the question of the innovative nature of Web 2.0 now that different examples have been discussed. To sum up the interim results until now:

- Blogs and wikis are not in the first way technological innovations. All examples for Web 2.0 use indicate that innovations take place more on the social level.

- Web 2.0 requires a different way of using already existing technologies as well as a large group of active users, with the technological environment becoming more and more intuitive and thus less restricted.

- There is a broad, heterogeneous and active group of users who require simple solutions to generate, administrate and publish content and who are motivated by the social prestige coming with it, the wish to interact with peers or the potential for freedom and democratisation movements.

- An active user base again requires corresponding internet access, access speed and reasonable prices.

On that basis we can return to the question posed above whether Web 2.0 services are 'first layer' or 'second layer' media (Kubicek, 1997) and say: if 'media' are understood as a social layer of mediated communication which describes the social aspects of creation and distribution of communication, all examples given have to be considered 'second layer media' (Kaletka and Pelka, 2010). One of their main characteristics is to establish social connection between their users (Kubicek, 1997, p.34). Again: while the development of user networks has been reported before 'Web 2.0' was labelled - Achim Bühl described the social impact of networking computers already in 1997 (p.167ff.) this used to be a phenomenon restricted to niches and special target groups. It was basically the contrary of a wide-spread users group.

The innovative aspect of Web 2.0 is the 'user-generated content' approach, which delegates the production and provision of content to the public. Compared to traditional media where editorial staff produces and distributes content, all Web 2.0 content is produced in a decentralised way while, in some cases, a group of editors may see to the rules being followed or moderate discussions.

Therefore, Web 2.0 can be described as a specific way of using the internet (a firstlayer medium) which takes advantage of this technological innovation and uses it to establish a new dimension of social utilisation (vividly described in the " 4 Cs of Social 
Media Framework', see Mishra, 2009). In short: the Web 2.0 comprises media that make use of the technological infrastructure 'internet' while adding a new social dimension by applying it collaboratively. Web 2.0 describes not only a modified utilisation but also a modified perception of the internet.

Now we will further elaborate how we define two basic elements of our Web 2.0 approach: the closely related concepts of 'content' and 'user innovation'. What is meant by 'content'? We understand content in a very broad way. It comprises every form of production, communication and participation. One example for content is the economic process of participation and 'peer production' described by Tapscott and Williams (2007, p.11).

The concept of 'user innovation' has been introduced in innovation research years ago. But in that case it primarily describes an enterprise-driven participation of users in product development (von Hippel, 2005; Flowers et al., 2009), not the individual and uncontrolled generation of content by users. The difference is crucial, because for enterprise-driven user innovation there are a number of options available to manage and control innovation, while Web 2.0 content is hardly controllable. The unpredictability of Web 2.0 innovations and the multitude of options for the users suggest the idea of Web 2.0 as a 'realm'. This realm provides possible courses of action which can be realised by the realm's numerous and heterogeneous 'inhabitants'.

It is remarkable in how many social sub-systems Web 2.0 is already .considered highly relevant. The speed of its diffusion is high. Until now, further diffusion has been realised through new groups of authors and commentators who have started using Web 2.0 and the corresponding increasing visibility and social acceptance of their products. But apart from the exploding number of users for well-established services such as Twitter, StayFriends or Wikipedia, Web 2.0 has also become subject of public funding, especially for capacity building projects.

One example: 'Telecentres' and telecentre networks promote the employability of target groups who face problems while entering the labour market. These telecentres receive public funding to build up and support online communities consisting of these target groups who would otherwise have no or limited access to internet-based communication. The promoters of this approach make use of Web 2.0-based online curricula to facilitate access to the world of labour (Kluzer and Rissola, 2009). The idea behind it is quite simple: it stems from projects in socially deprived districts in South American countries and is now being implemented in many countries of the European Union. Through funding of technological equipment, the development of learning methodology and curricula, local networking and the qualification of the so-called 'facilitators' not only internet access is being offered to vulnerable target groups, but also a directed and guided access to Web 2.0 services which are supposed to qualify the users.

The 'facilitators', or mediators, play a central role in basically all local telecentre projects: they support the users and ideally have to have wide-spread competences, they act as guides for using the Web 2.0 services and therefore have to be qualified pedagogically, they have to be guides for internet and Web 2.0, they serve as counsellors and finally as local networkers. They have contacts to job centres, schools, regional enterprises and other actors to actively support the integration of their students into the world of labour. Of course, this is an ideal qualification profile of a Web 2.0 telecentre facilitator. A facilitator curriculum is currently being developed in an European project the authors are involved in, called "Vocational training for e-inclusion facilitators", funded in the Leonardo da Vinci programme. 
This is one very concrete example how traditional social sectors (such as qualification and adult learning) can benefit from Web 2.0 services: they offer additional functions (qualification and access to the labour market) for specific target groups (in this case: people in a local community who are excluded from the world of labour and partly from social life as a whole). Wiki-based curricula, in this case, can help to implement cooperative and participative learning which is also characterised by a low-threshold access.

More generally speaking: for Germany, the survey 'Zukunft und Zukunftsfähigkeit der deutschen Informations- und Kommunikationstechnologie ' ('Future and sustainability of the German information and communication sector', delivered by Münchener Kreis et al., 2008) focuses the social impact of Web 2.0 in a number of fields. In the chapter dedicated to Web 2.0, which they consider primarily as a technology, they underline its potential to bridge the digital gap in society. Quite remarkably, they address the biggest potential to bridge technological illiteracy to technology. In reference to the results of Kluzer and Rissola (2009), the basic argument is valid: if we succeed in even further lowering technological access requirements - e.g. by adequately qualifying pedagogic staff in telecentres - Web 2.0 services become more and more suited for target groups with low technological and media expertise.

\section{Challenge: what is new about user-generated content?}

In the same way, Dery (1996) considered the communication culture of the internet as a logical technology-based development further promoting the discussion culture born in the $1960 \mathrm{~s}$, one could argue that user-generated content - which is the central aspect of the here developed definition of Web 2.0 - is not bound to digital media. One could call Samizdat literature, political flyers and letters to the editor or even wall paintings as usergenerated content. These forms of publication comply with two of the criteria set by the OECD definition for user-generated content. They reflect a 'certain amount of creative effort', which is 'created outside of professional routines and practices'. So, is usergenerated content a unique benefit of the Web 2.0?

No, it is not. The ECREA Section 'Communication History' dedicated the conference 'User-Generated Content. Historical Perspectives on the Participation of Audiences in Social Communication' to that question (see: http:/www.dgpuk.de/index.cfm?id=3756). Different, yet unpublished, papers pointed out the function of user-generated content in 19th century political movements, modern - but offline - religious events and even the political discussion in the renaissance Venice. In these examples, content - provided by private persons and aimed at the public - created effects on public discourses, opinions and decisions. In this understanding, user-generated content is bound to neither special technologies nor an innovation at all.

But our perspective remains clear: the combination of the internet technology (as a technical innovation or 'first-layer' medium) with the utilisation by a large and heterogeneous public, created the Web 2.0 . Web 2.0 for us is a social innovation that has the potential to influence the way people work, communicate and participate. Furthermore, it fits the upcoming challenges of modern information society with work that has to be fast, cooperative, incremental and dialogue based. 


\section{References}

Alby, T. (2007) Web 2.0. Konzepte, Anwendungen, Technologien, Hanser,'München.

Buhl, A. (1997) Die virtuelle Gesellschaft. Ökonomie, Politik und Kultur im Zeichen des Cyberspace, Westdeutscher Verlag, Opladen.

Dery, M. (1996) Cyber. Die Kultur der Zukunft, Verlag Volk \& Welt, Berlin.

Diaz, A. and Rissola, G. (2009) 'e-Facilitating networking in distance co-operation contexts', in Franz, H-W. and Sarcina, R. (Eds.): Building Leadership in Project and Network Management, Springer, Berlin, pp.249-2.54.

Flowers, S., Sinozic, T. and Patel, P. (2009) Prevalence of user innovation in the EU. Analysis based on the Innobarometer Surveys of 2007 and 2009. Available online at: http://www. proinno-europe.eu/admin/uploaded_documents/EIS_2009_User_innovation.pdf (accessed on 26 January 2010).

Howaldt, J. and Schwarz, M. (2010) 'Social innovation: concepts, research fields and international trends', in Henning, K. and Hees, F. (Eds.): Studies for Innovation in a Modern Working Environment - International Monitoring, Vol. 5, Eigenverlag, Aachen. Available online at: http:/www.sfs-dortmund.de/odb/Repository/Publication/Doc\%5C1289\%5CIMO Trendstudie_Howaldt_Schwarz_englische_Version.pdf (accessed on 9 June 2011).

Howaldt, J., Kopp, R. and Schwarz, M. (2009) 'Shaping Innovation by Social Research - the New Role of the Social Sciences', in Harrisson, D., Szell, G. and Bourque, R. (Eds.): Social Innovation, the Social Economy and World Economic Development, Peter Lang, Frankfurt, pp.245-260.

Howaldt, J., Kopp, R. and Schwarz, M. (2008) 'Innovationen (forschend) gestalten. Zur neuen Rolle der Sozialwissenschaften', WSI-Mitteilungen, 2/2008, pp.63-69.

Kaletka, C. and Bastian, P. (2010) 'Web 2.0 zwischen technischer und sozialer Innovation: Anschluss an die medientheoretische Debatte', in Howaldt, J. and Jacobsen, H. (Eds.): Soziale Innovation. Auf dem Weg zu einem postindustriellen Innovationsparadigma, VS Verlag, Wiesbaden, pp.143-161.

Kluzer, S. and Rissola, G. (2009) 'E-inclusion policies and initiatives in support of employability of migrants and ethnic minorities in Europe', Information technologies \& International Development, Vol. 5, No. 2, 67-76.

Kubicek, H. (1997) Bürgerinformation durch 'neue' Medien? Analysen und Fallstudien zur Etablierung elektronischer Informationssysteme im Alltag, Westdeutscher Verlag, Opladen.

Leskovec, J., Backstrom, L. and Kleinberg, J. (2009) Meme-tracking and the Dynamics of the News Cycle. School of Computer Science, Carnegie Mellon University, Pittsburgh PA. Available online at: http://www.cs.cmu.edu/ jure/pubs/quotes-kdd09.pdf (26 January 2010).

Maaß, C. and Pietsch, G. (2007) Web 2.0 als Mythos, Symbol und Erwartung. Diskussionsbeitrag der Fakultăt fur Wirtschaftswissenschaft der Fernuniversităt in Hagen.

Mishra, G. (2009) The $4 \mathrm{Cs}$ social media framework. Available online at: http://www. gauravonomics.com/blog/the-4cs-social-media-framework/ (9 June 2011).

Münchener Kreis e. V., Deutsche Telekom AG, TNS Infratest GmbH and European Center for Information and Communication Technologies (EICT) GmbH (Eds) (2008) Zukunft \& Zukunftsfähigkeit der deutschen Informations- und Kommunikationstechnologie. Abschlussbericht der ersten Projektphase. Available online at: http://www.downloadtelekom.de/dt/StaticPage/59/97/56/081205_zukunftsstudie.pdf_599756.pdf (26 January 2010).

Neuberger, C., Nuernbergk, C. and Rischke, M. (2007) 'Weblogs und Journalismus; Konkurrenz, Ergänzung oder Integration? Eine Forschungssynopse zum Wandel der Öffentlichkeit im Internet', Media Perspektiven, No. 2, pp.96-112.

Pelka, B. (Ed) (2008) Das Prinzip Wiki in der Praxis: Theorie, Anwendung, Anleitung, Der Andere Verlag, Tönning/Lubeck/Marburg. 